\title{
Ebola Risk and Preparedness: A National Survey of Internists
}

\author{
Ishani Ganguli, $M D^{1,2}$, Yuchiao Chang, $P h D^{1,2}$, Arlene Weissman, PhD ${ }^{3}$, Katrina Armstrong, $M D^{1,2}$, \\ and Joshua $P$. Metlay, MD, $P h D^{1,2}$
}

${ }^{7}$ Massachusetts General Hospital, Boston, MA, USA; ${ }^{2}$ Harvard Medical School, Boston, MA, USA; ${ }^{3}$ American College of Physicians, Philadelphia, PA, USA.

BACKGROUND: The 2014-2015 Ebola virus disease (Ebola) epidemic centered in West Africa highlighted recurring challenges in the United States regarding risk communication and preparedness during global epidemics.

OBJECTIVE: To investigate perceptions, preparedness, and knowledge among U.S. internists with regard to Ebola risk.

DESIGN: Cross-sectional Web-based national survey distributed by e-mail between December 2014 and January 2015.

PARTICIPANTS: Practicing U.S. internists participating in a research panel representative of American College of Physicians (ACP) membership.

MAIN MEASURES: Respondents' perceptions of Ebola, reported sources of information, and reported management of possible Ebola cases. The primary predictor was the possibility of encountering Ebola (based on respondents' geographic proximity to designated airports or confirmed Ebola cases, or on their patients' travel histories). Pre-specified outcomes included reported management intensity in clinical vignettes involving patients at low risk of symptomatic Ebola as well as reported Ebola preparedness.

KEY RESULTS: The survey response rate was $46.1 \%$. Among the 202 respondents, $9.9 \%$ (95 \% CI 6.2-14.9\%) reported that they had recently evaluated a patient who had traveled to West Africa. Seventy percent (95\% CI 63.0-76.0 \%) reported a practice-level protocol. The Centers for Disease Control and Prevention (CDC) was the most popular source for Ebola information (75.2\%, 95 $\%$ CI 68.7-81.0\%). Most respondents felt very (45.0\%) or somewhat prepared (52.0\%) to communicate information about or diagnose Ebola, especially those with the possibility of encountering Ebola and those who reported medical journals, professional groups, or government as information sources. One-fifth of respondents (19.8\%, $95 \%$ CI 14.5-26.0\%) reported overly intensive management for low-risk patients. Those with the possibility of encountering Ebola were less likely to report overly intensive management (3.1 vs. $22.9 \%, p=0.011$ ).

CONCLUSIONS: Internists had wide-ranging views and understanding of Ebola risk; those least likely to

Electronic supplementary material The online version of this article (doi:10.1007/s11606-015-3493-1) contains supplementary material, which is available to authorized users.

Received May 4, 2015

Revised July 7, 2015

Accepted July 27, 2015

Published online August 20, 2015 encounter Ebola were most likely to be overly aggressive in managing patients at low risk. Our findings underscore the need for better risk communication through various information channels to empower frontline providers in infectious disease outbreaks.

KEYWORDS: Ebola; risk communication; physician behavior; medical decision making; evidence based medicine.

J Gen Intern Med 31(3):276-81

DOI: $10.1007 / \mathrm{s} 11606-015-3493-1$

(C) Society of General Internal Medicine 2015

\section{INTRODUCTION}

The impact of the 2014-2015 outbreak of Ebola virus disease (Ebola) was deeply felt in West Africa, with more than 27,000 cases and 11,000 deaths in Guinea, Liberia, and Sierra Leone as of the time of publication. ${ }^{1}$ In the United States, where there have been four locally diagnosed cases and one fatality to date, the potential domestic impact of the disease received a great deal of public health and media attention, at times to deleterious effect. ${ }^{2,3}$ Following missteps in management of the first locally diagnosed case in Dallas, which led to loss of trust in health officials, ${ }^{3,4}$ several state governors initiated quarantines of even symptom-free international aid workers returning from Ebola relief efforts, ${ }^{5}$ and there were several high-profile news reports of institutions asking students and employees with spurious connections to the disease to stay home ${ }^{6-8}$

Among health care practitioners, there was evidence of heightened concern as well. Transmission of the Ebola virus occurs only by direct contact with the bodily fluids of symptomatic individuals, and the typical incubation period ranges from 2 to 21 days - evidence that is the basis for guidelines issued by the CDC. ${ }^{9}$ Yet of the 650 inquiries to the CDC about suspected Ebola cases between July and mid-November 2014, $75 \%$ referenced individuals who had not recently traveled to a country with Ebola or been in contact with an Ebola patient. Furthermore, only $18 \%$ were deemed potential cases based on initial signs, symptoms, and risk factors. ${ }^{10}$ In several instances, pursuing Ebola pathways may have delayed clinicians' efforts to confirm the true diagnosis.

The U.S. experience with the Ebola epidemic is reminiscent of prior outbreaks, such as avian flu and severe acute respiratory syndrome (SARS), in which public and health professional reactions were poorly matched to communicated risks, ${ }^{6,11}$ yet factors contributing to these disconnects are not 
well understood. The West African Ebola outbreak offers a unique opportunity to investigate how communication infrastructure and personal factors affect the ability of frontline health care providers to respond appropriately to global health crises. Our goal was to measure how the risks of Ebola were perceived and acted upon by health professionals, particularly in ambulatory care settings, where preparedness is uniquely challenging. ${ }^{12}$ In addition, we wanted to explore the role that different information sources and regional context played in shaping provider attitudes and knowledge. To address these aims, we conducted a national survey of U.S. internists, examining self-reported preparedness among physicians and whether their potential for clinical exposure to Ebola was predictive of their management approach in hypothetical clinical scenarios.

\section{METHODS}

We conducted a Web-based national survey of U.S. internists between December 4, 2014, and January 20, 2015. The survey was conducted in collaboration with the American College of Physicians (ACP) and covered the domains of physician and practice characteristics, risk perception, information sources, preparedness, and knowledge related to Ebola.

\section{Study Population}

The ACP Research Center surveyed member physicians who agreed to participate in its nationally representative panel established in June 2011. After excluding medical students, affiliate members, honorary fellows, and non-U.S. members, the ACP used stratified random sampling to create the Internal Medicine Insider Research Panel. ${ }^{13}$ The panel is regularly adjusted to represent membership across multiple demographic characteristics. Panelists agree to participate in an average of two projects per month, and are rewarded for completing each survey with points redeemable for gift cards.

Within this panel, we selected all self-reported general internists and geriatricians who practiced primary care, and excluded retired physicians, residents, clinical fellows, and clinicians who self-reported less than $25 \%$ of their time in direct patient care. We identified 462 physicians (39\% of the panel) who met these pre-established criteria for survey administration.

\section{Survey Instrument}

The 23-item survey was developed based on a literature review of comparable viral outbreaks. ${ }^{14,15}$ The instrument included five sections. The first section captured demographic and practice characteristics and assessed physicians' exposure to patients at risk for Ebola. The second section assessed physicians' perceptions of Ebola risk. The third section asked respondents to rate the personal importance of information sources and their level of preparation in order to identify and manage possible cases. The fourth section measured how physicians assessed the risk of Ebola versus influenza in a series of clinical scenarios, which were written such that influenza was more likely in each case while the probability of Ebola varied. Finally, the survey posed clinical vignettes testing knowledge of appropriate management of suspected Ebola, transmission methods, and incubation period based on CDC guidelines circulating at the time of survey distribution. ${ }^{9}$ The survey was field-tested by primary care physicians at two adult internal medicine clinics affiliated with Massachusetts General Hospital; insights from the field testers' survey responses and oral comments were incorporated into the final instrument (eSurvey).

\section{Survey Distribution}

The survey was distributed via e-mail to 462 panel members on December 4, 2014, and remained in the field for 48 days. Five repeat requests were sent via e-mail to non-responders in order to improve the response rate.

\section{Measures}

Our pre-specified primary predictor was the possibility of encountering Ebola, a binary measure meant to approximate a provider's pre-test probability of diagnosing Ebola in his/her patients. Possibility of encountering Ebola was defined as either self-report of seeing patients who had traveled to West Africa or been exposed to the Ebola virus, or geographic proximity to a designated U.S. port of entry for flights from Ebola-affected West African countries (John F. Kennedy International Airport, Newark Liberty International Airport, Dulles International Airport, Chicago O'Hare International Airport, and Hartsfield-Jackson Atlanta International Airport) or to a confirmed Ebola case (Dallas, TX; New York City, NY). Geographic proximity was defined by whether a respondent's ZIP code was included in the combined statistical area of an index city.

Our primary outcome was level of management intensity, which was defined a priori by responses to two of the clinical vignettes (eSurvey 3-4) evaluating knowledge of transmission methods and incubation period in patients with low likelihood of contagious Ebola virus disease. In both vignettes, an overly intense management response based on CDC guidelines was defined as choosing to isolate the patient, test for Ebola, or call the local hospital. A moderateintensity response was defined as choosing to ensure health department monitoring (this was guideline-concordant for question $3 \mathrm{~A}$ ), and a low-intensity response was defined as choosing reassurance/routine care (this was guidelineconcordant for questions $3 \mathrm{~B}$ and 4 ). The more intensive of the two vignette responses defined the respondent's intensity level. Our secondary outcomes were self-reported preparedness to diagnose cases and communicate risk as well as the presence of a protocol for Ebola diagnosis and treatment at the respondent's practice site. 


\section{Data Analysis}

We reported respondent demographic and practice characteristics, perceptions of Ebola risk, information sources, preparedness for Ebola, and approach to managing Ebola risk. Descriptive statistics included frequency counts and percentages for categorical variables, and ranges and means with standard deviations for continuous variables.

We examined associations between outcomes and predictors including provider demographics, practice characteristics, self-reported information sources, and geographic regions.

Both management intensity and Ebola preparedness were considered as ordinal variables, with three response categories (low/moderate/overly intense, and very/somewhat/not at all, respectively). Having an Ebola protocol was dichotomized into yes vs. no/not sure. In bivariate analyses, we compared groups using Mantel-Haenszel chi-square tests for ordinal outcomes and chi-square tests for dichotomized outcomes. We conducted a proportional odds ordinal regression model to examine the effect of the possibility of encountering Ebola on management intensity while controlling for age, sex, race, and practice setting. There was no evidence of violating the proportional odds assumption based on the score test. All analyses were performed using SAS version 9.4 statistical software (SAS Institute Inc., Cary, NC, USA). All reported $p$ values are two-tailed, and $p<0.05$ was considered statistically significant.

We submitted our proposal to the Partners Institutional Review Board and it was granted exemption status. The study had no external funding source.

\section{RESULTS}

\section{Demographic and Practice Characteristics}

We received 213 completed questionnaires from among 462 potential respondents, for a response rate of $46.1 \%$ (eFigure). Survey responses were excluded for 11 respondents who reported that they spent no time delivering primary care. Respondents and non-respondents were similar based on demographic features, including gender and age. Among the 202 respondents who were eligible and who completed the survey, age ranged from $28-83$ years, with a mean of 52.5 years (10.6); $35.6 \%$ were women (Table 1$)$.

Ten respondents $(5.0 \%, 95 \%$ CI $2.4-8.9 \%)$ reported that they had worked in an international medical relief effort, while $4.0 \%$ (95\% CI 1.7-7.7\%) stated that they had considered engaging in Ebola relief efforts in West Africa. Twenty respondents $(9.9 \%, 95 \%$ CI 6.2-14.9\%) reported that they had seen at least one patient within the last 3 months who had traveled to West Africa in 2014, and one participant $(0.5 \%, 95$ $\%$ CI $0.0-2.7 \%$ reported seeing a patient who had been exposed to Ebola. Thirty-two physicians $(15.8 \%, 95 \% \mathrm{CI}$ $11.1-21.6 \%$ ) met the previously described definition of having the possibility of encountering Ebola.
Table 1 Demographic and Practice Characteristics of Respondents $(N=\mathbf{2 0 2})$

\begin{tabular}{lc}
\hline \hline Characteristic & $\boldsymbol{N} \mathbf{( \% )}$ \\
\hline Age group (years) & \\
Under 40 & $29(14.4)$ \\
$40-55$ & $85(42.1)$ \\
$56+$ & $88(43.6)$ \\
Sex & \\
Male & $124(61.4)$ \\
Female & $72(35.6)$ \\
Not stated & $6(3.0)$ \\
Race & $144(71.3)$ \\
White & $48(23.9)$ \\
Nonwhite & $10(5.0)$ \\
Not specified & \\
Time spent in direct patient care & $37(18.3)$ \\
$<50 \%$ & $22(10.9)$ \\
$50-74 \%$ & $143(70.8)$ \\
$>75 \%$ & \\
Practice type & $31(15.3)$ \\
Solo practice & $81(40.1)$ \\
Group private practice* & $44(21.8)$ \\
Academic medical center & $34(16.8)$ \\
Community/government practice & $12(5.9)$ \\
Other & \\
Practice setting & $79(39.1)$ \\
Urban & $22(10.9)$ \\
Rural & $98(48.5)$ \\
Suburban & $3(1.5)$ \\
Other & $10(5.0)$ \\
Worked in international medical relief effort & $32(15.8)$ \\
Possibility of encountering Ebola & \\
\hline
\end{tabular}

There were no missing data

* Group private practice includes practices not affiliated with an academic medical center

\section{Perception of Ebola Risk}

Nearly two-thirds of respondents either agreed or strongly agreed $(66.3 \%, 95 \%$ CI $59.4-72.8 \%)$ that they accepted the risk of contracting Ebola as part of their job. Nine percent (9.4\%, $95 \%$ CI 5.8-14.3\%) expressed fear of contracting Ebola, $15.8 \%$ (95 \% CI 11.1-21.6 \%) stated that their physician colleagues had this fear, and $37.1 \%$ (95\% CI 30.5$44.2 \%$ ) indicated that the fear was present among their nonphysician colleagues (Fig. 1).

\section{Information Sources on Ebola}

The CDC was the most popular self-reported source for Ebola information $(75.2 \%, 95 \%$ CI 68.7-81.0 \%) (Table 2), with $23.8 \%$ of respondents $(95 \%$ CI $18.1-30.2 \%$ ) listing it as their top source. Eighty-eight percent $(88.1 \%, 95 \%$ CI $82.8-$ $92.2 \%$ ) reported having reviewed CDC Ebola guidelines at least once during the preceding 12 months, compared to $73.8 \%$ (95 \% CI 67.1-79.7 \%) who reviewed guidelines on influenza and $57.4 \%$ (95\% CI 50.3-64.3\%) who reviewed guidelines on sexually transmitted infections over the same period. Following the CDC, the next most common sources were the respondent's employer and the Internet (18.3 and $11.9 \%$, respectively). When information sources were collapsed into four major categories, $66.3 \%$ (95\% CI 59.4$72.8 \%$ ) of respondents chose journals, $83.2 \%$ (95 \% CI $77.3-$ $88.1 \%$ ) chose professional sources (employer or professional 


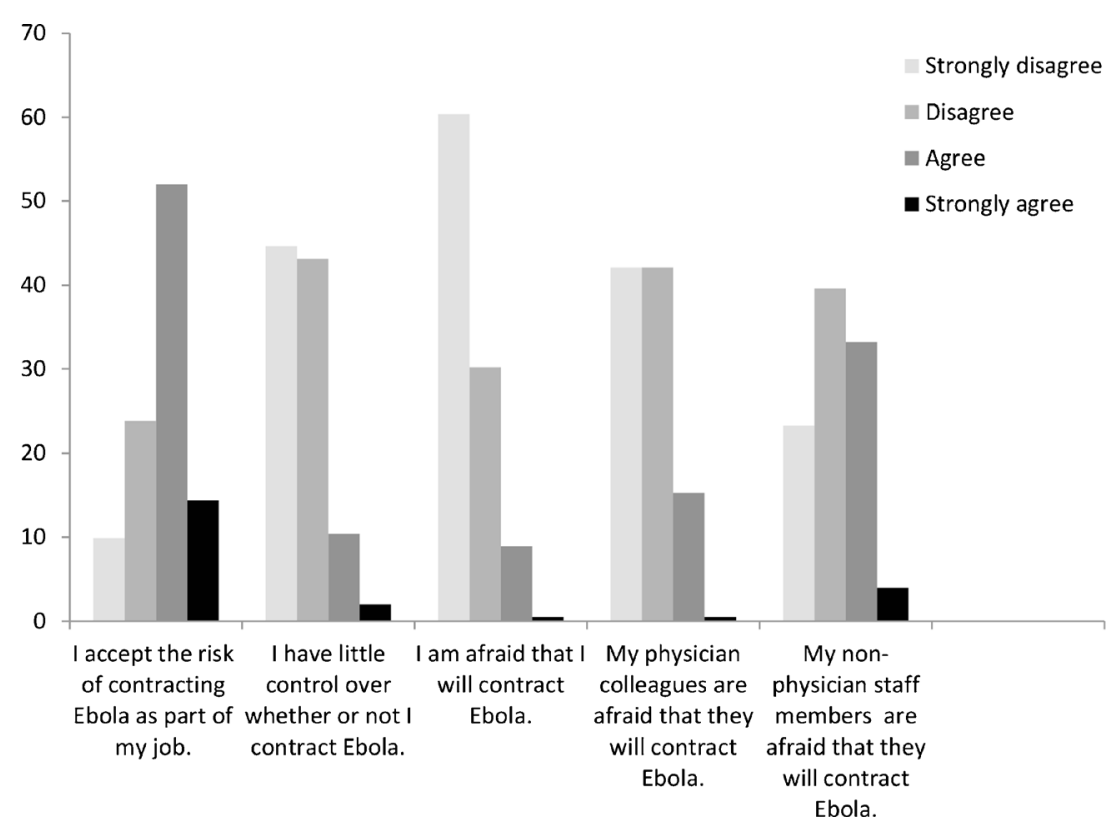

Fig. 1 Ebola risk perception. The figure shows the percentage of physicians who provided each response. There were no missing data.

organization), $81.7 \%$ (95 \% CI 75.6-86.8 \%) chose government sources (state/local government, CDC, international health agency), and $75.2 \%$ (95\% CI 68.7-81.0\%) chose the lay press (TV, newspaper or magazine, radio, Internet).

\section{Ebola Preparedness}

Almost all respondents stated that they felt very prepared $(45.0 \%)$ or somewhat prepared $(52.0 \%)$ to communicate the risk of acquiring Ebola to a patient. Similarly, most respondents felt very prepared $(27.2 \%)$ or somewhat prepared $(68.3 \%)$ to identify possible cases (eTable). Seventy percent (95\% CI 63.0-76.0\%) reported a protocol in place at their practice site to identify and manage possible cases.

Predictors of Preparedness. Doctors were more likely to report that they were very prepared to communicate Ebola risk to a patient if they had a possibility of encountering Ebola ( 68.8 vs. $40.6 \%, p=0.003$ ) or if they reported medical journals (54.5 vs. $26.5 \%, p<0.001$ ), government (49.1 vs. $27.0 \%$,

Table 2 Self-Reported Sources of Information on Ebola Used in the Last 3 Months $(N=\mathbf{2 0 2})$

\begin{tabular}{llr}
\hline \hline Information source & & $\boldsymbol{N} \mathbf{( \% )}$ \\
\hline Lay press & TV News & $111(55.0)$ \\
& Internet & $108(53.5)$ \\
& Newspaper or magazine & $85(42.1)$ \\
Journals & Radio & $51(25.2)$ \\
Professional sources & Medical journals & $134(66.3)$ \\
& Professional organization & $126(62.4)$ \\
Government sources & Employer & $105(52.0)$ \\
& CDC & $152(75.2)$ \\
& State/local government & $84(41.6)$ \\
& International health agency & $31(15.3)$ \\
\hline
\end{tabular}

There were no missing data. CDC Centers for Disease Control and Prevention $p=0.030$ ), or professional groups ( 48.8 vs. $26.5 \%, p=0.032$ ) as information sources on Ebola compared to those not choosing each of these sources. Physicians with a possibility of encountering Ebola were more likely to report they were very prepared to identify possible Ebola cases (40.6 vs. $24.7 \%, p=0.033)$. Similarly, physicians who reported using medical journals as information sources were more likely to report they were very prepared to identify possible Ebola cases compared to those who did not ( 31.3 vs. $19.1 \%, p=0.006$ ). Doctors from solo practices were less likely to report a protocol for Ebola diagnosis and treatment $(38.7 \%, 95 \% \mathrm{CI}$ $21.8-57.8 \%)$ than those from group private practices $(63.0 \%$, $95 \%$ CI 51.5-73.4\%), academic medical centers ( $95.5 \%, 95$ $\%$ CI 84.5-99.4\%), or community/government practices $(76.5 \%, 95 \%$ CI $58.8-89.3 \%)(p<0.001)$. Respondents with a possibility of encountering Ebola were not more likely to report a protocol (78.1 vs. $68.2 \%, p=0.26$ ).

Fifty-eight percent (57.9 \%, $95 \%$ CI 50.8-64.8\%) of physicians reported that they had changed or were planning to change their practice to manage the risk of Ebola. Among them, $24.8 \%$ (95 \% CI 17.3-33.6\%) had spent more than ten hours on Ebola preparedness (Table 3).

\section{Approach to Managing Risk of Ebola}

Respondents were given a series of clinical scenarios involving a patient with flu-like symptoms presenting in November, 2014, and were asked to choose whether Ebola or influenza was the more likely diagnosis. Respondents were unlikely to choose Ebola $(3.0 \%, 95 \%$ CI 1.1-6.4\%) if there was no additional information, if the patient reported a cough and sore throat ( $0.5 \%, 95 \%$ CI $0-2.7 \%$ chose Ebola), or if the patient was a local emergency nurse $(1.0 \%, 95 \%$ CI $0.1-3.5 \%$ chose 
Table 3 Practice Changes among Physicians Endorsing Current or Planned Changes $(N=117)$

\begin{tabular}{lc}
\hline \hline Practice change & N (\%) \\
\hline $\begin{array}{l}\text { Have front desk staff screen all patients for Ebola risk } \\
\text { factors }\end{array}$ & $83(70.9)$ \\
$\begin{array}{l}\text { Have front desk staff screen all patients who call in with } \\
\text { flu-like symptoms }\end{array}$ & $63(53.8)$ \\
$\begin{array}{l}\text { Have office staff isolate patients who screen positive for } \\
\text { Ebola risk factors }\end{array}$ & $85(72.6)$ \\
$\begin{array}{l}\text { Stop taking new patients from countries that have been } \\
\text { affected with Ebola }\end{array}$ & $2(1.7)$ \\
$\begin{array}{l}\text { Stop scheduling return visits for patients from countries } \\
\text { that have been affected with Ebola }\end{array}$ & $4(3.4)$ \\
$\begin{array}{l}\text { Require office staff to stay home if they develop flu-like } \\
\text { symptoms consistent with Ebola in the absence of any }\end{array}$ & $20(17.1)$ \\
known exposure & \\
$\begin{array}{l}\text { Purchase the specific personal protective equipment } \\
\text { necessary for protection from Ebola }\end{array}$ & $38(32.5)$ \\
\hline
\end{tabular}

There were no missing data

Ebola). In contrast, most physicians chose Ebola as more likely if the patient had direct contact with an asymptomatic Ebola patient $(62.4 \%, 95 \%$ CI $55.3-69.1 \%)$ or had volunteered in an Ebola treatment center the previous month $(85.1 \%, 95 \%$ CI $79.5-89.8 \%)$. Nearly a quarter of respondents $(23.8 \%, 95 \%$ CI 18.1-30.2\%) chose Ebola if the patient had recently returned from South Africa.

For each of the clinical vignettes (eSurvey 2-4), most participants provided responses concordant with $\mathrm{CDC}$ guidelines: When asked about evaluating a patient with flu-like symptoms in clinic, $91.6 \%$ (95\% CI 86.9-95.0\%) reported they would ask for a travel history first. When asked about managing an asymptomatic international aid worker who had returned from Ebola containment efforts, $69.3 \%$ (95 \% CI 59.3-78.1\%) of those who were told she had returned 10 days prior said they would confirm health department monitoring. Among those who were told she had returned 25 days prior, $51.5 \%$ (95\% CI 41.3-61.6\%) said they would proceed with preventive care. More than half of respondents ( $58.4 \%, 95 \%$ CI $51.3-65.3 \%$ ) said they would offer reassurance to a nurse who reported that there was a patient with Ebola in her hospital with whom she had no direct contact. Based on their responses regarding Ebola incubation period and transmission (eSurvey 3-4), $19.8 \%$ of physicians ( $95 \%$ CI 14.5-26.0 \%) were categorized as reporting overly intense management, while $56.4 \%$ (95\% CI $49.3-63.4 \%)$ reported moderate-intensity management and $23.8 \%$ (95\% CI 18.1-30.2\%) low-intensity management, as previously defined.

Predictors of Management Intensity. The possibility of encountering Ebola was significantly inversely associated with intensity of management. Among physicians with a possibility of encountering Ebola, $3.1 \%$ chose overly intense management, while $62.5 \%$ chose moderate-intensity and $34.4 \%$ chose low-intensity management strategies. In contrast, $22.9 \%$ of physicians without a possibility of encountering Ebola chose overly intense management, while $55.3 \%$ chose moderate-intensity and $21.8 \%$ chose low-intensity management strategies $(p=0.011)$. In ordinal logistic regression adjusting for age, sex, race, and practice setting, respondents with a possibility of encountering Ebola had lower odds of choosing the more intensive management strategy (OR 0.41, $95 \%$ CI $0.19-0.87, p=0.02$ ). There was no statistically significant association between management intensity and age, sex, race, top information source, practice type, or practice setting.

\section{DISCUSSION}

The 2014-2015 Ebola outbreak centered in West Africa left United States public health officials, health systems, and the media struggling at times to mount a measured response. This raised important recurring questions about how we manage information to help frontline clinicians in times of public health crisis. For this reason, we investigated internists' perceptions and knowledge of Ebola using a national survey.

While few respondents said that they had seen patients who had recently traveled to West Africa, most reported consulting CDC guidelines on managing Ebola at least once; the CDC was the most frequently cited information source. Almost all respondents gave the guideline-concordant answer on obtaining a travel history, but there was considerable variation in responses regarding incubation period and transmission risk. When we looked at management intensity of these responses, we found that the only significantly associated factor was the possibility of encountering Ebola: physicians who practiced outside metropolitan areas associated with Ebola cases or designated airports, or who did not report patients with recent West African travel, were more likely to endorse overly aggressive management. This may be because these physicians were less motivated to stay abreast of Ebola clinical guidelines or due to a lack of institutional or community infrastructure to support the physicians in management - although we found no association between the possibility of encountering Ebola and the presence of an institutional protocol. It is not clear whether this is a physician- or institution-level phenomenon, though there was no association with practice setting or type.

There was wide variation in the number of respondents reporting that Ebola was more likely than influenza in a series of scenarios. Notably, recent contact with an asymptomatic Ebola patient, the presence of vomiting and diarrhea, and even travel to South Africa swayed many respondents toward Ebola as the more likely pathogen. Reassuringly, a Medscape survey of health professionals found that $69 \%$ ranked influenza as a high threat to public health, while only $17 \%$ had this view of Ebola. ${ }^{16}$

Despite variation among respondents in Ebola guideline knowledge, we found that almost all physicians felt at least somewhat prepared to communicate the risk of acquiring Ebola and to identify possible cases - especially if they used informational sources other than the lay press or had a possibility of encountering Ebola. In addition, most reported a 
protocol in place at their practice site, though only $39 \%$ of solo practitioners reported such a protocol, and many had personally changed their practice accordingly. These results are consistent with those of the Medscape survey, in which $63 \%$ of health professionals reported that they were prepared to treat Ebola and $97 \%$ reported confidence in their knowledge of Ebola. ${ }^{16}$ Finally, while few respondents reported a fear of contracting Ebola, a greater number reported such a fear among their physician colleagues, and nearly half reported the fear among non-physician staff members - which may reflect differences in education level ${ }^{17}$ or the effect of physicians choosing more socially desirable opinions for themselves.

Overall, despite most respondents reporting that they had reviewed CDC guidelines, our results confirm a disconnect between physicians' confidence in Ebola preparedness and their knowledge of appropriate diagnosis and management. ${ }^{10}$. This raises important questions about how to engage doctors in providing appropriate care beyond guideline distribution-perhaps through outreach by professional groups, electronic decision support, or government-mandated training. Given the popularity of lay press sources (Table 2), it will also be critical to address limitations in the quality of health journalism. ${ }^{18,19}$

This investigation had several limitations. We achieved a $46 \%$ response rate, suggesting the possibility of response bias, though demographic characteristics were similar between respondents and non-respondents. Physicians who self-selected to participate may have had greater knowledge about Ebola or were more comfortable with Ebola preparedness activities, so our study may overestimate physician confidence, knowledge, and preparedness. While the study population was representative of membership in the nation's largest internal medicine organization, it may not represent all U.S. internists. We do not know whether physicians would act in real life as they reported in hypothetical scenarios. This is a cross-sectional survey, so we cannot show causality. We acknowledge that the constructs used for possibility of encountering Ebola and fear were not externally validated, though they had face validity and were based on literature regarding similar epidemics.

Future studies might investigate the reasons behind individual management decisions and the cost and clinical impact of delays or compromise of routine care due to misplaced concern about Ebola or a comparable outbreak. We demonstrated that knowledge, risk perception, and preparedness are influenced by individual practice experiences and location. As global health threats continue to emerge, improved communication of health risks and appropriate management strategies are needed.

Contributors: We thank Stephen Calderwood, MD (Massachusetts General Hospital) for his insightful thoughts on our study approach; Steven Weinberger, MD (American College of Physicians) for his guidance and support of our study design; and Erika Shenoy, $M D, P h D$ (Massachusetts General Hospital) for her thoughtful review of our manuscript.
Funders: No financial or material support was provided for the design or conduct of the study; collection, management, analysis, or interpretation of the data; or preparation, review, or approval of the manuscript.

Prior Presentations: This work has not been presented at any conference.

Conflict of Interest: The authors declare that they do not have a conflict of interest.

Corresponding Author: Ishani Ganguli, MD; Massachusetts General Hospital, 55 Fruit Street Bulfinch 205, Boston, MA 02114, USA (e-mail: iganguli@partners.org).

\section{REFERENCES}

1. Centers for Disease Control and Prevention. 2014 Ebola Outbreak in West Africa-Case Counts. http://www.cdc.gov/vhf/ebola/outbreaks/2014west-africa/case-counts.html. Accessed June 17, 2015.

2. Luckerson V. Fear, Misinformation, and Social Media Complicate Ebola Fight. Time. Oct. 8. 2014. http://time.com/3479254/ebola-social-media/. Accessed February 24, 2015.

3. Sharfstein J. On fear, distrust, and Ebola. JAMA. 2015;313:784.

4. Rosenbaum L. Communicating uncertainty-Ebola, public health, and the scientific process. N Engl J Med. 2015;372:7-9.

5. Drazen JM, Kanapathipillai R, Campion EW, et al. Ebola and quarantine. N Engl J Med. 2014;371:2029-30.

6. Carey B. EXPERTS OFFER STEPS for Avoiding Public Hysteria, a Different Contagious Threat. New York Times. 2014 Oct 15. http://www.nytimes. com/2014/10/16/health/ebolas-other-contagious-threat-hysteria.html. Accessed February 24, 2015.

7. Giordano R, Fichera A. Maple Shade parents struggle with Ebola fears. The Philadelphia Inquirer. October 22, 2014. http://articles.philly.com/ 2014-10-22/news/55284980_1_ebola-fears-school-nurse-suspected-ebola. Accessed February 4, 2015.

8. Hartmann M. Kentucky Teacher Resigns Over Parents' Dumb Ebola Fears. New York Magazine. 2014 Nov 4. http://nymag.com/daily/intelligencer/2014/11/kentucky-teacher-resigns-over-dumb-ebola-fears.html. Accessed February 4, 2015.

9. Centers for Disease Control and Prevention. Ebola (Ebola Virus Disease). http://www.cdc.gov/vhf/ebola/index.html. Accessed February 24, 2015.

10. Karwowski MP, Meites E, Fullerton KE, et al. Clinical inquiries regarding Ebola virus disease received by CDC-United States, July 9-November 15, 2014. MMWR Morb Mortal Wkly Rep. 2014;63:1175-9.

11. Ovadia KL, Gazit I, Silner D, Kagan I. Better late than never: a reexamination of ethical dilemmas in coping with severe acute respiratory syndrome. J Hosp Infect. 2005;61:75-9.

12. Wu HM, Fairley JK, Steinberg J, Kozarsky P. The potential Ebolainfected patient in the ambulatory care setting: preparing for the worst without compromising care. Ann Intern Med. 2015;162:66-7.

13. Internal Medicine Insider Research Panel. https://iminsider.org/. Accessed July 20, 2015.

14. Imai T, Takahashi $\mathbf{K}$, Hasegawa N, Lim MK, Koh D. SARS risk perceptions in healthcare workers, Japan. Emerg Infect Dis. 2005; 11:404-10.

15. Wong TY, Koh GC, Cheong SK, et al. A cross-sectional study of primarycare physicians in Singapore on their concerns and preparedness for an avian influenza outbreak. Ann Acad Med Singap. 2008;37:458-64.

16. Goodman B. Survey Reveals Concerns Over Ebola. WebMD Health News. 2014 Oct 31. http://www.webmd.com/news/20141031/survey-revealsconcerns-over-ebola. Accessed January 3, 2015.

17. Harvard School of Public Health and SSRS. Ebola Poll. August 13-17, 2014. http://www.hsph.harvard.edu/news/press-releases/poll-findsmany-in-us-lack-knowledge-about-ebola/. Accessed January 29, 2015.

18. Koenig KL, Schultz CH. The 2014 Ebola Virus Outbreak and Other Emerging Infectious Diseases. Preliminary Electronic Draft. https://www. acep.org/uploadedFiles/ACEP/practiceResources/issuesByCategory/ publichealth/The\%202014\%20Ebola\%20Virus\%20Outbreak.pdf. Accessed June 22, 2015.

19. Schwitzer G, Mudur G, Henry D, et al. What are the roles and responsibilities of the media in disseminating health information? PLoS Med. 2005;2(7):e215. 